\title{
Visión estratégica sobre el desarrollo de la ganadería en Brasil
}

\section{Strategic vision for the development of livestock in Brazil}

\author{
Bernardino de Carvalho Thiago ${ }^{1}$, Hurtado Nery Víctor Libardo ${ }^{2}$ y \\ Torres Novoa Diana Milena ${ }^{3}$ \\ ${ }^{1}$ Investigador CEPEA/ESALQ/USP, Brasil; ${ }^{2}$ MVZ. MSc. PhD. Docente Unillanos y \\ ${ }^{3} \mathrm{MVZ}$, MSc, Profesor Universidad de los Llanos, Colombia \\ vhurtado@unillanos.edu.co
}

Recibido 16 de Mayo 2014, Aceptado 03 de Septiembre 2014

\section{RESUMEN}

En este texto se presenta como ha sido el mercado de carnes, el impacto en la economía brasilera y se compara la situación a nivel mundial. Dado el peso económico de la producción bovina en el mercado internacional se hace énfasis en el desarrollo de la ganadería en Brasil, a partir de políticas públicas relacionadas con la producción pecuaria y apoyo a la industria frigorífica. En conclusión, el futuro del mercado de carnes y el agronegocio brasileiro es promisorio dada la disponibilidad de tierras y agua existentes en el país, haciendo inversión en capital humano, tecnologías e infraestructura.

Palabras clave: Costo de producción, industria cárnica, producción bovina.

\begin{abstract}
This text is presented as it was the meat market, the impact on the Brazilian economy and the situation is compared globally. Given the economic importance of livestock productions in the international market whit emphasis on livestock development in Brazil, from public policies related to livestock production and support the meat industry. In conclusion, the future of the meat market and agribusiness in Brazil is promising given the availability of land and water existing in the country, making investment in human capital, technology and infrastructure.
\end{abstract}

Keywords: Cost of production, meat industry, beef production. 


\section{INTRODUCCIÓN}

Al analizar el sector pecuario, en el mercado de la carne se debe revisar con cuidado lo referente a los indicadores económicos locales y mundiales, puesto que, en los últimos años, principalmente a partir del segundo semestre de 2008, la economía global atraviesa por una grave crisis financiera que causa estragos en los mercados globalizados, unos con más fuerza y otros como Brasil con menor relevancia. Esa crisis hace que los flujos de dinero disminuyan, lo que impacta negativamente en el comercio y la producción de riqueza del país. La Figura 1 muestra la evolución del producto interno bruto (PIB) en los países emergentes y en desarrollo, en los de economías avanzadas y el promedio mundial.

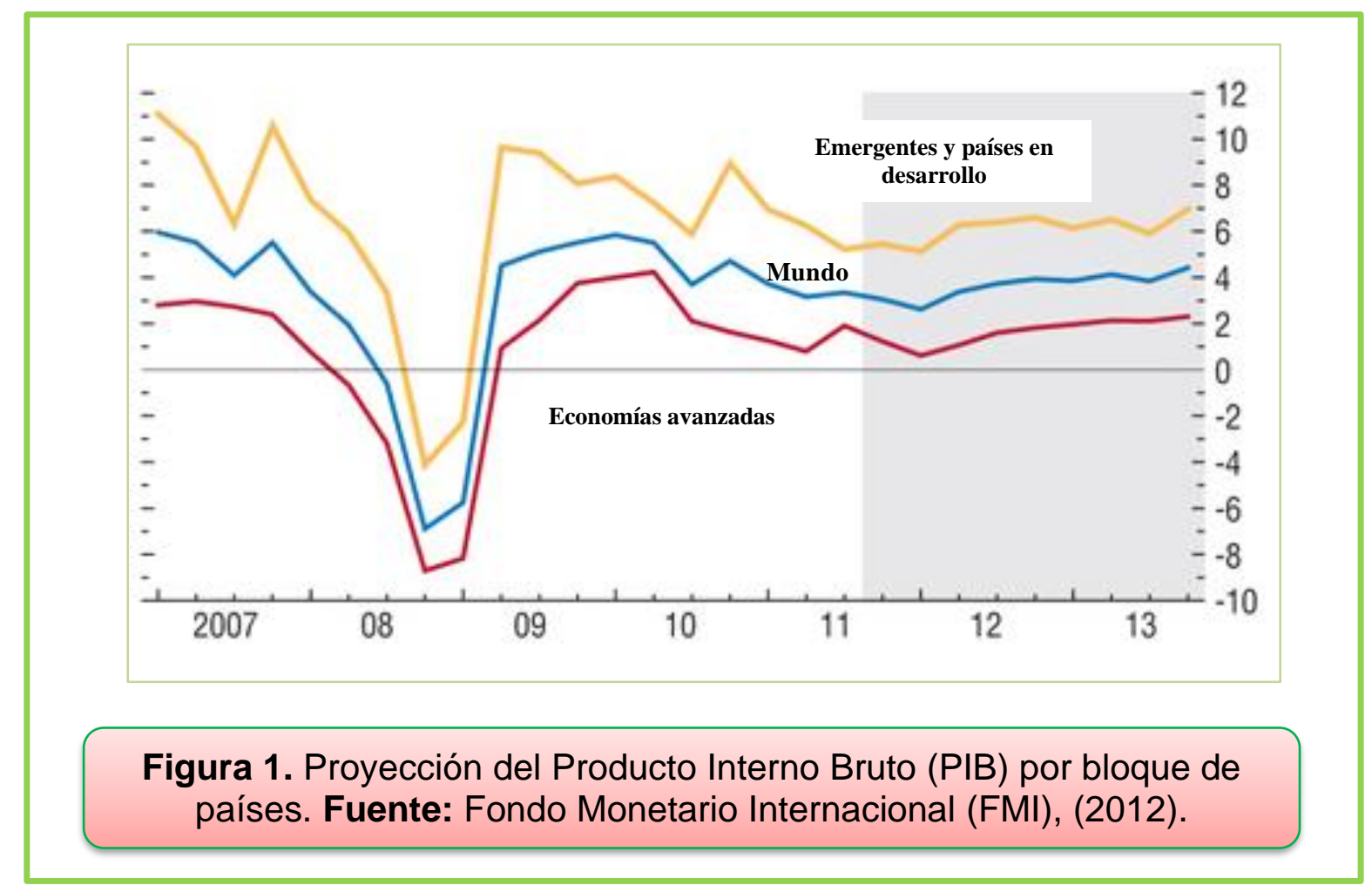

Se puede observar que hubo impacto de la crisis en todo el mundo, sin embargo, los países que consiguieron mantener un nivel positivo de producción fueron los que están en desarrollo, lo contrario sucedió con los países ricos como USA, Japón y la Zona Europea que pasan por una situación financiera caótica demostrada por las tasas de deuda pública y por el desempleo que aumenta 
mensualmente, principalmente en los dos últimos años, lo que obliga a la necesidad de solicitar créditos financieros de otros países.

A mediano y largo plazo la expectativa mundial es que el incremento económico sea liderado por los países emergentes, entre ellas el grupo BRIC (Brasil, Rusia, India y China) como se muestra en la Figura 2, puesto que el crecimiento de la riqueza del BRIC impulsará el aumento del consumo.

Como se mencionó anteriormente, en los últimos años la economía brasileira ha estado en el pico más alto aplicando políticas fuertes, lo que favorece el crecimiento del país, en indicadores socioeconómicos mostrando esa tendencia, con el menor nivel de desempleo en los últimos años, cerca de 6\%. El PIB, es creciente en aproximadamente cuatro trillones de reales $(R \$)$ que aunado a la inflación controlada, impulsan la economía, el crecimiento y consumo doméstico de Brasil.

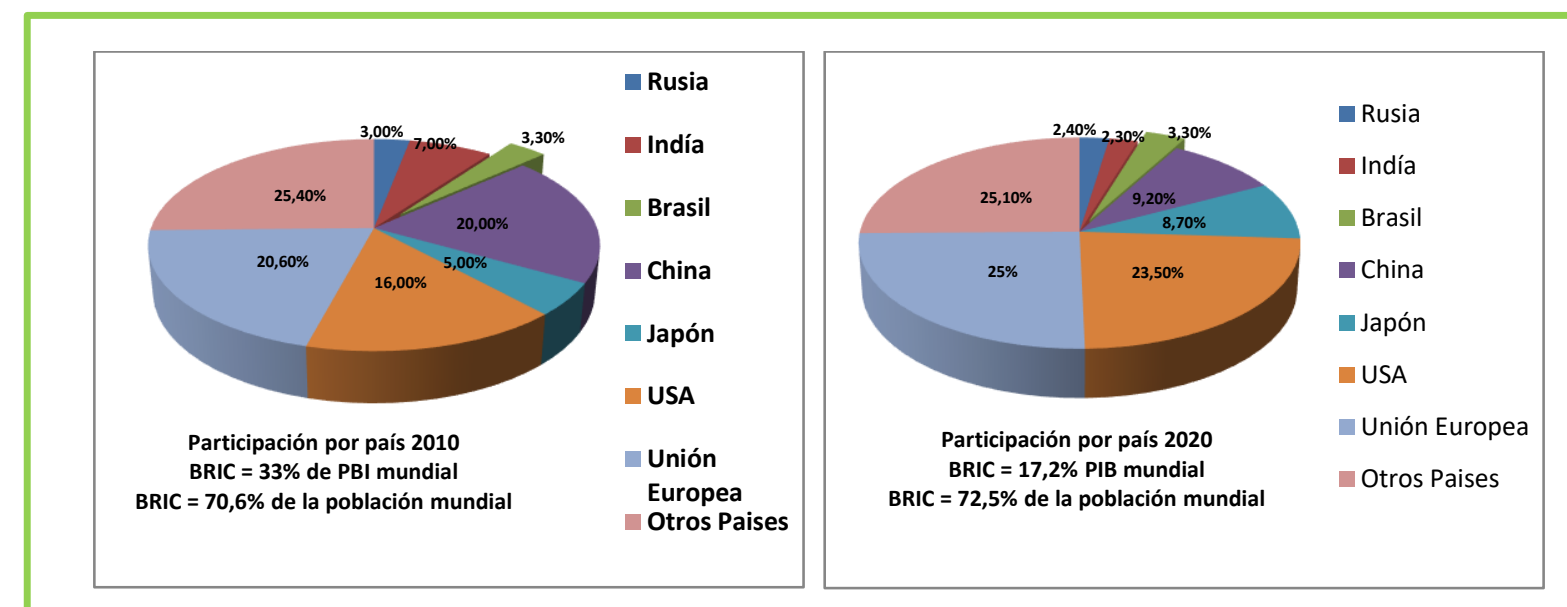

Figura 2. Proyección de la participación por país del PIB (\%) y población del BRIC. Fuente: FMI e FAO, (2011).

Un hecho importante de los últimos años es la disminución de la pobreza y el surgimiento de la nueva clase media, lo que ocasiona un incremento de la demanda de proteína animal, principalmente por carne bovina. En los últimos cinco años la proporción de personas en la clase social C subió más de $60 \%$ (Figura 3), lo que implica una mayor demanda, entre tanto las clases A y B 
exigirán productos de mejor calidad, lo que ratifica el momento favorable de la economía brasilera.

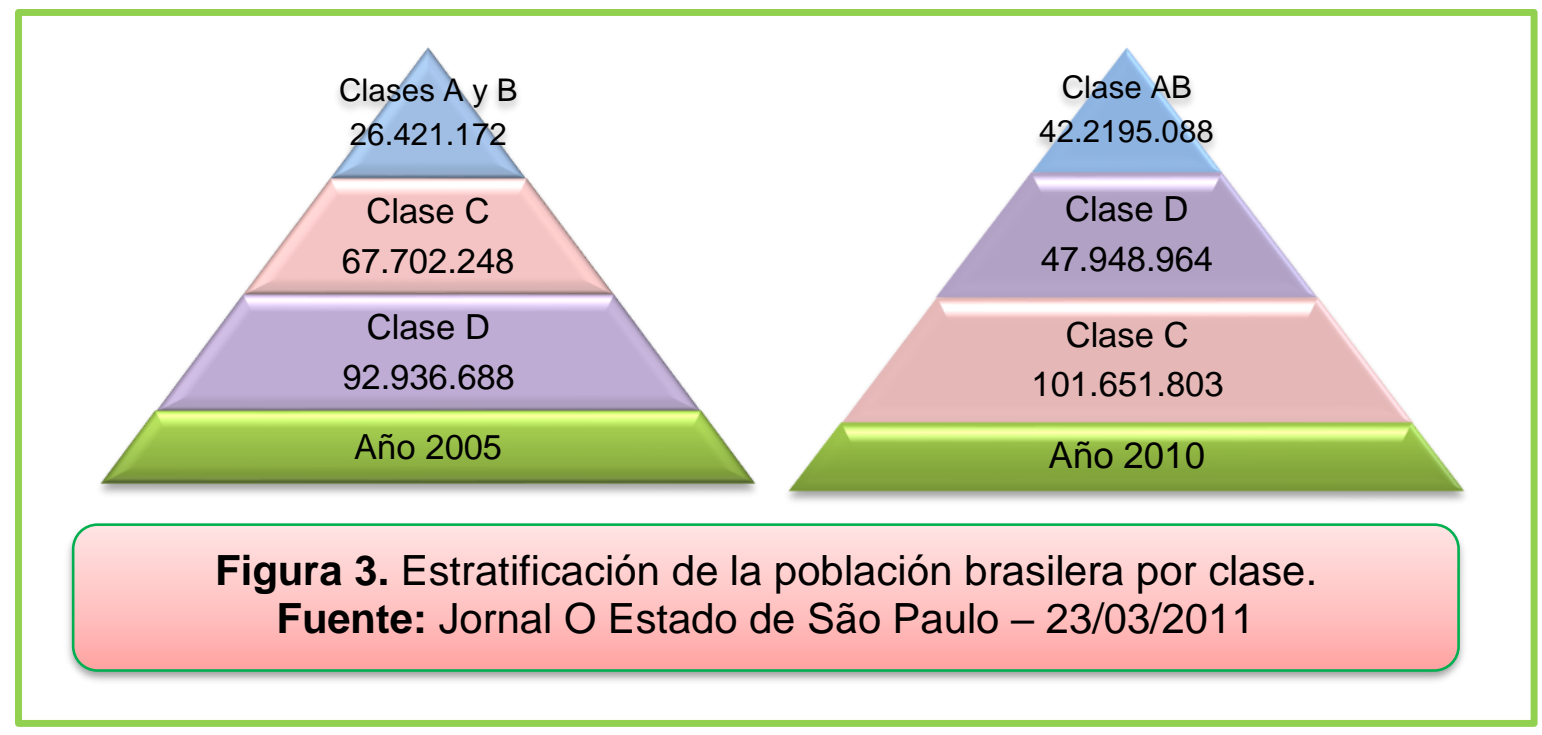

Después del 2004, el volumen de consumo de carne (Figura 4) creció fuertemente superando los $\mathrm{R} \$ 40$ billones por año, cifra que muestra la importancia del sector para la economía y la producción, que fue posible por el "plano real" y medidas de control de la inflación, intereses y crédito a la población. Después del plan económico en 1994, hubo crecimiento en el consumo interno de alimentos, con el aumento de la población y de la renta, lo que propició un incremento sustancial en la producción nacional de carnes, es importante destacar que es directamente proporcional al PIB de un país, porque lleva a un aumento de la demanda de carne y viceversa. Por eso, el impacto de la crisis produjo inestabilidad para este mercado en el ambiente mundial, que al contrario en Brasil, por la solidez de la economía fue amenizado posibilitando el desarrollo del sector productivo de proteína animal.

\section{EL MERCADO BRASILERO DE CARNES}

A pesar de ser considerado un país en desarrollo, el consumo de carnes en Brasil se ubica en niveles observados en las naciones más ricas, superando la cifra de $100 \mathrm{~kg} /$ habitante / año. 


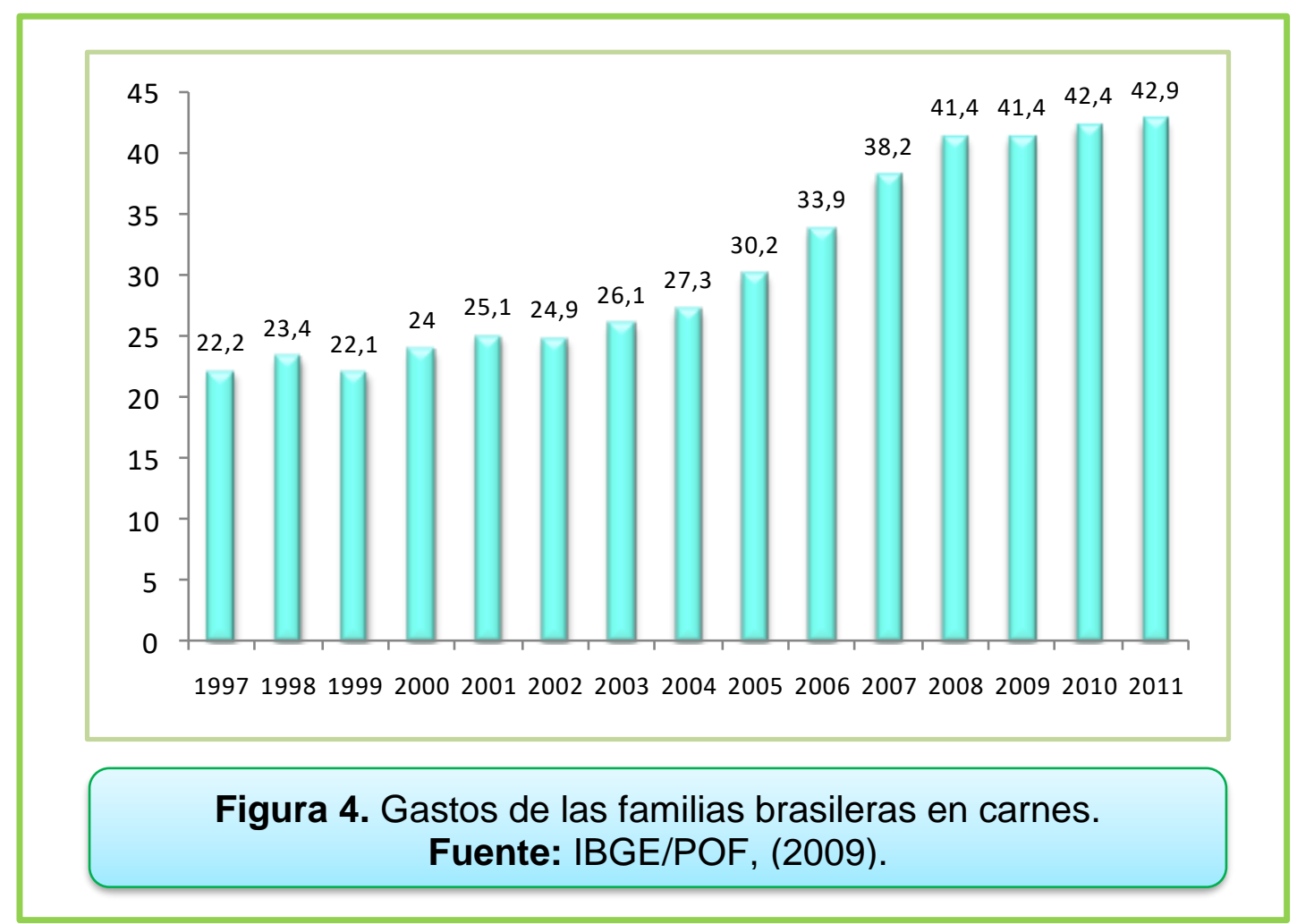

Hasta los años 70, la carne bovina representaba más del $50 \%$ del total de carnes consumido por los brasileros, en segundo lugar la carne de cerdo y el pollo en tercera posición. A partir de los años 80 , la búsqueda de alimentación saludable, hace que el consumo de carnes consideradas blancas aumente. En la década actual, la carne de pollo superó a la bovina en la dieta de los brasileros, consumiendo en promedio $44 \mathrm{~kg}$ de pollo y la carne de cerdo fue relegada al tercer lugar con $14 \mathrm{~kg} /$ año por persona (Figura 5). El aumento del consumo de pollo en relación con las otras está asociado al precio relativamente menor.

Brasil posee un potencial grande en el mercado interno del consumo de carne, ejemplo, la de cerdo, la menos consumida, aun así, tiene un mercado para ser conquistado y expandido, puesto que se busca igualar al consumo de los países desarrollados, aproximadamente $70 \mathrm{~kg} / \mathrm{habitante} / \mathrm{año}$.

El ingreso real de los brasileros creció entre 1960 y 1990, según Barros y Mendoça, (1995) posibilitando el aumento del consumo de alimentos, en los últimos años, con este mismo escenario con dificultades para conquistar e 
incrementar ventas en el mercado exterior por barreras sanitarias, el desarrollo del mercado interno es considerado de primordial importancia para el crecimiento del comercio de carnes.



Martins, (1998) reitera que el consumo de alimentos y de otros bienes está determinado por factores económicos, sociales, culturales y sus interrelaciones, siendo el primero el más importante por precios de los propios bienes, de los complementarios y sustitutos, que se relaciona con el nivel de ingreso de la población. Otro factor que puede influenciar, es lo que destacan Pinazza y Araújo, (1993) afirmando que el aumento del ingreso hace que la participación de cereales en la dieta disminuya mientras el consumo carnes aumenta.

Un coeficiente de elasticidad es importante en el análisis del aumento del ingreso y demanda, observando un menor valor en el comportamiento de mercado de pescado en los diferentes estratos, en comparación al caso de la carne bovina de primera, con una elasticidad media, lo que quiere decir que en caso de que el ingreso aumente $10 \%$, hay un incremento en los gastos de $6.23 \%$ para adquirir 
carne bovina de primera, con lo que demuestra que este tipo de carne producto sufre fuerte influencia de una buena economía y consecuentemente ingresos altos por parte de los brasileros (Tabla 1).

Tabla 1. Coeficientes de elasticidad-ingreso del gasto per cápita con diferentes tipos de carne (POF 2008/09)

\begin{tabular}{|c|c|c|c|c|}
\hline \multirow{2}{*}{ Tipo de carne } & \multicolumn{3}{|c|}{ Elasticidad - estrato } & \multirow{2}{*}{$\begin{array}{l}\text { Elasticidad } \\
\text { media }\end{array}$} \\
\hline & $\mathbf{I}$ & II & III & \\
\hline Bovina de primera & 0,726 & 0,535 & 0,064 & 0,623 \\
\hline Bovina de segunda & 0,323 & 0,114 & $-0,034$ & 0,169 \\
\hline Cerdo & 0,623 & 1,088 & $-0,157$ & 0,627 \\
\hline Pollo & 0,139 & $-0,005$ & 0,304 & 0,128 \\
\hline Pescado & 0,006 & $-0,206$ & 0,498 & 0,125 \\
\hline Leche & 0,540 & 0,530 & 0,378 & 0,486 \\
\hline
\end{tabular}

Fuente: Carvalho, (2011)

El aumento de la demanda por carnes beneficia toda la cadena, lo cual se comprueba mediante el valor bruto de la producción (VBP) en 2010 donde se observa la importancia que el sector cárnico tiene para la economía nacional, cuando la carne bovina generó un VBP de $R \$ 43.170$ millones, la de pollo $R \$$ 20.903 millones y la de cerdo $\mathrm{R} \$ 8.361$ millones, para un total de los tres sectores de $R \$ 72.435$ millones para la economía brasilera (Confederação da Agricultura e Pecuária do Brasil - CNA, 2011).

\section{CADENA DE PRODUCCIÓN DE CARNE BOVINA}

La ganadería bovina de carne está presente en el escenario económico nacional, desde la época colonial, en las últimas décadas, se desarrolló a través de la expansión de la frontera agrícola, con la incorporación de nuevas tierras, siendo la mayoría desprovista de infraestructura y con degradación del suelo por el sistema intensivo de producción de granos, caracterizándose la producción nacional siempre por el sistema extensivo, cambiando este panorama en los últimos años, con la incorporación de nuevas tecnologías que buscan el aumento de la productividad de los sistemas intensivos en algunas regiones, llamados de confinamiento o semiconfinamiento. 
En los años 90 e inicio del nuevo siglo, se observa una ganadería nacional con altos índices de productividad y una industria nacionalizada concentrada en grupos de acción interregional con una producción de carne dispersa por todo el territorio nacional, con la mayor concentración en la región centro-sur del país, siendo el $45 \%$ del hato nacional, donde se ubican los mejores rebaños en los estados de Mato Grosso, Minas Gerais, Mato Grosso do Sul y Goiás. En relación a los precios pagados al productor, durante muchos años, el bovino gordo en Brasil fue considerado como reserva de valor, debido a la alta liquidez y por la ociosidad del precio (Figura 6), pero en 1994, después del Plan Real (control de la inflación) los precios de los animal han permanecido constantes, lo que obligó a todos los agentes del mercado a trabajar con márgenes más restringidos, es decir a profesionalizar y a trabajar mejor la gestión de la actividad.

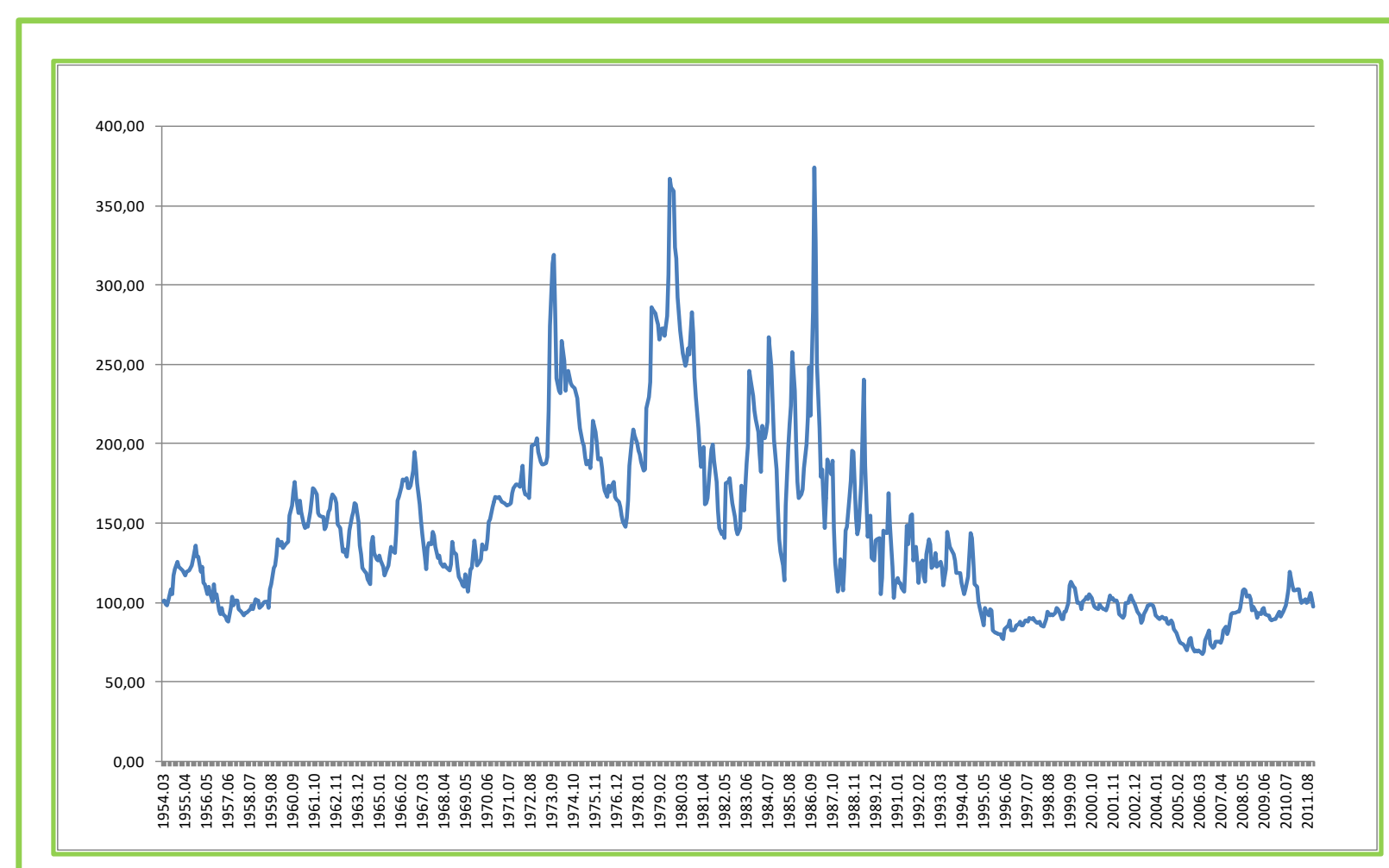

Figura 6. Fluctuación del precio anual de la carne de bovino gordo en $R \$ / 15 \mathrm{~kg}$ en Brasil de 1954 a 2011. Fuente: CEPEA, (2012). 
En el mercado internacional es notorio el crecimiento de la participación brasilera con carne bovina, a partir del final de los 90, en función de la profesionalización de la actividad, rígido control de enfermedades, bajo costo, calidad del producto y desvalorización cambiaria, se puede observar en el 2004, que Brasil se convierte en el mayor exportador de esta carne, posición que se mantiene en la actualidad.

El mayor mercado de carne nacional era la Unión Europea, hasta el año 2007, por la crisis de la fiebre aftosa de 2005 en Brasil y por las barreras impuestas por la comunidad a las haciendas brasileras en los años siguientes, hicieron que otros países ganaran importancia para las ventas de la carne brasilera, como Rusia, Hong Kong, Egipto e Irán recientemente. El mercado europeo, es muy importante para la carne brasilera (Figura 7) debido a los buenos precios que se pagan por ella. Sin embargo, en este momento de escasez de dinero hay preocupación por la cantidad comprada por los europeos, siendo en 2011 Rusia el principal destino de la carne nacional, otros mercados como Hong Kong, Egipto e Irán tuvieron un importante crecimiento.



Figura 7. Evolución de las exportaciones de carne bovina (toneladas) para los principales compradores. Fuente: Abiec, (2012). 
El mercado externo fue muy importante para el desarrollo de la industria nacional, siendo el potencial productivo un factor esencial para atraer inversionistas internacionales, en los años recientes, las crecientes barreras comerciales hicieron que esos grupos perdieran interés por Brasil y la industria se nacionalizó, pero el patrón internacional de la industria permaneció.

La industria brasilera en los últimos años trabaja con márgenes reducidas de mercado en comparación a veinte años atrás (Figura 8), lo que muestra la necesidad de la eficiencia en la producción y comercialización, puesto que se requiere que el sector industrial trabaje con el sacrificio y deshuesado, ya que la carne consigue más valor agregado para la empresa.

Con la crisis financiera de 2008, hubo reducción del crédito internacional y el encarecimiento del dinero prestado por el mercado, ocasionó que varios grupos solicitaran su recuperación por la vía judicial y hasta reconocer su estado de quiebra y ser comprados por otras empresas, el gobierno afrontó dicha situación a través de préstamos del Banco Nacional de Desarrollo (BNDES), lo que jugó un papel fundamental en la ayuda a algunas industrias. A finales de 2010 había en el país cerca de 180 frigoríficos habilitados para vender carne al mercado internacional, de los cuales 39\% estaban en manos de cuatro grandes grupos, siendo responsables del $33 \%$ del sacrificio de animales con un total de 80.773 animales sacrificados por día.

Estos cuatro grandes grupos de la industria frigorífica, poseían unidades de sacrificio en Uruguay, Argentina, Paraguay, USA y Australia, principalmente con el objetivo de expandir nuevos mercados y buscar alternativas para aumentar sus negocios, la cual tuvo como principal pilar, al gobierno brasilero a través del BNDES y los Fondos de Pensión. Estas organizaciones tienen acciones de esos frigoríficos (Tabla 2), en total el gobierno Brasilero posee cerca de US\$ 7 billones de esas empresas, sin ese dinero la industria cárnica no tendría condiciones de expansión y consolidación en el mercado local e global. 


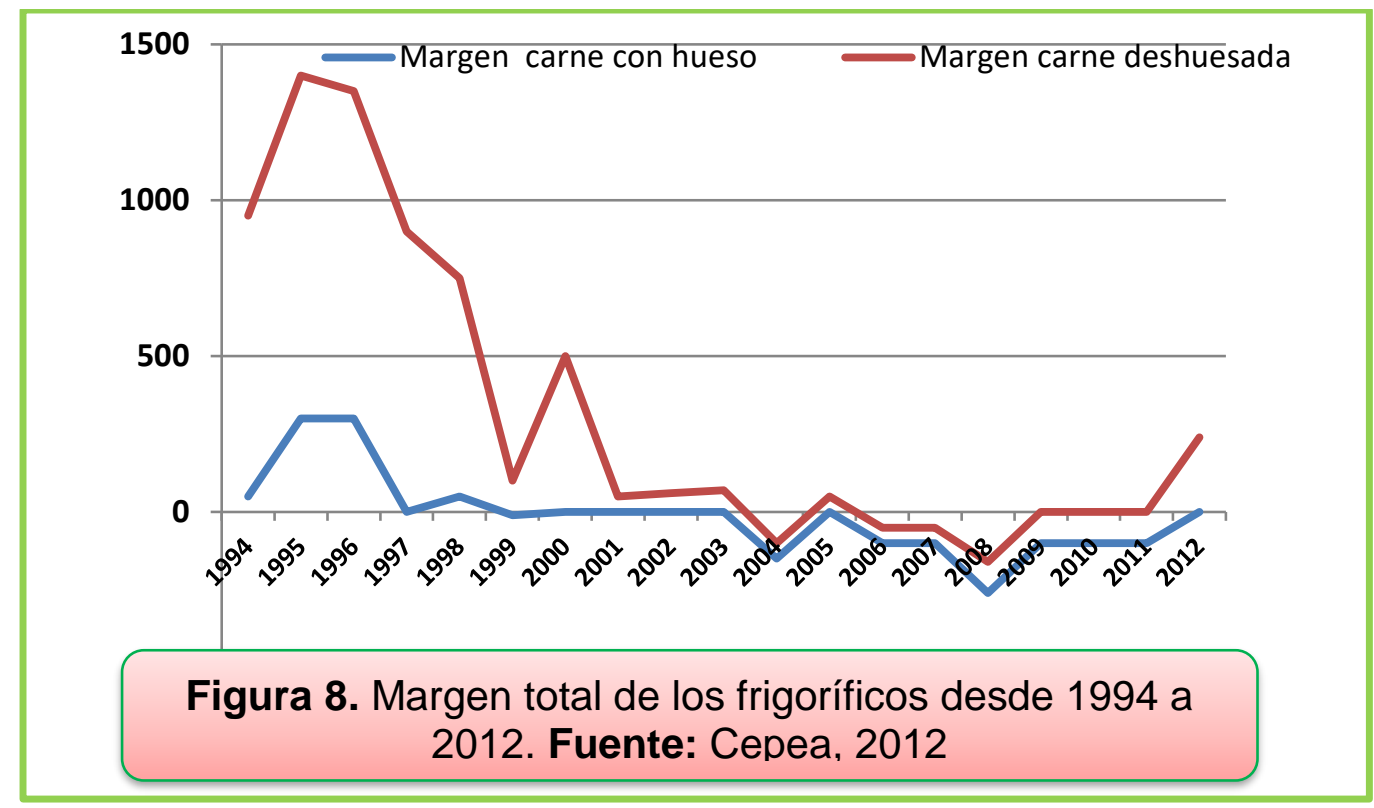

Tabla 2. Participación del gobierno brasilero en las acciones de los grupos

\begin{tabular}{cccccc} 
Empresa & $\begin{array}{c}\text { Gobierno } \\
\%\end{array}$ & Inversionistas & $\begin{array}{c}\text { Número de } \\
\text { acciones }\end{array}$ & $\begin{array}{c}\text { Valor } \\
\text { R\$ }\end{array}$ & Total R\$ \\
\hline JBS & 31,4 & BNDESPAR & 931.069 .588 & 7.25 & 6.750 .254 .513 \\
MINERVA & 12,7 & Previ - BB & 110.846 .320 & 33.81 & 3.747 .714 .079 \\
BRFOODS & 10,04 & Petros & 87.560 .126 & 33.81 & 2.960 .407 .860 \\
MARFRIG & 13,9 & BNDES & 48.200 .827 & 9.85 & 474.778 .146 \\
& & Total & & & $\mathbf{1 3 . 9 3 3 . 1 5 4 . 5 9 8}$ \\
\hline
\end{tabular}

Fuente: JBS, MINERVA, MARFRIG, BRFOODS, 2012.

${ }^{*}$ Banco Nacional de Desarrollo

\section{COSTO DE PRODUCCIÓN}

El gran desafío de la ganadería en los últimos años ha cobrado importancia debido a los gastos en alimentación y mano de obra, porque se ha aumentado la productividad del hato principalmente controlando y optimizando sus gastos, así compitiendo con cultivos, como caña de azúcar. Se observa la evolución del costo de producción y de ingresos del ganadero brasilero desde 2004 en los cuales, durante siete años, fueron superiores (106.8\%) a los ingresos $(69.3 \%)$, lo que indica descapitalización de la mayoría de los productores, cerca del $28 \%$ de los gastos están relacionados con la mano de obra y $23 \%$ de sal mineral (Figuras 8 y $9)$. 


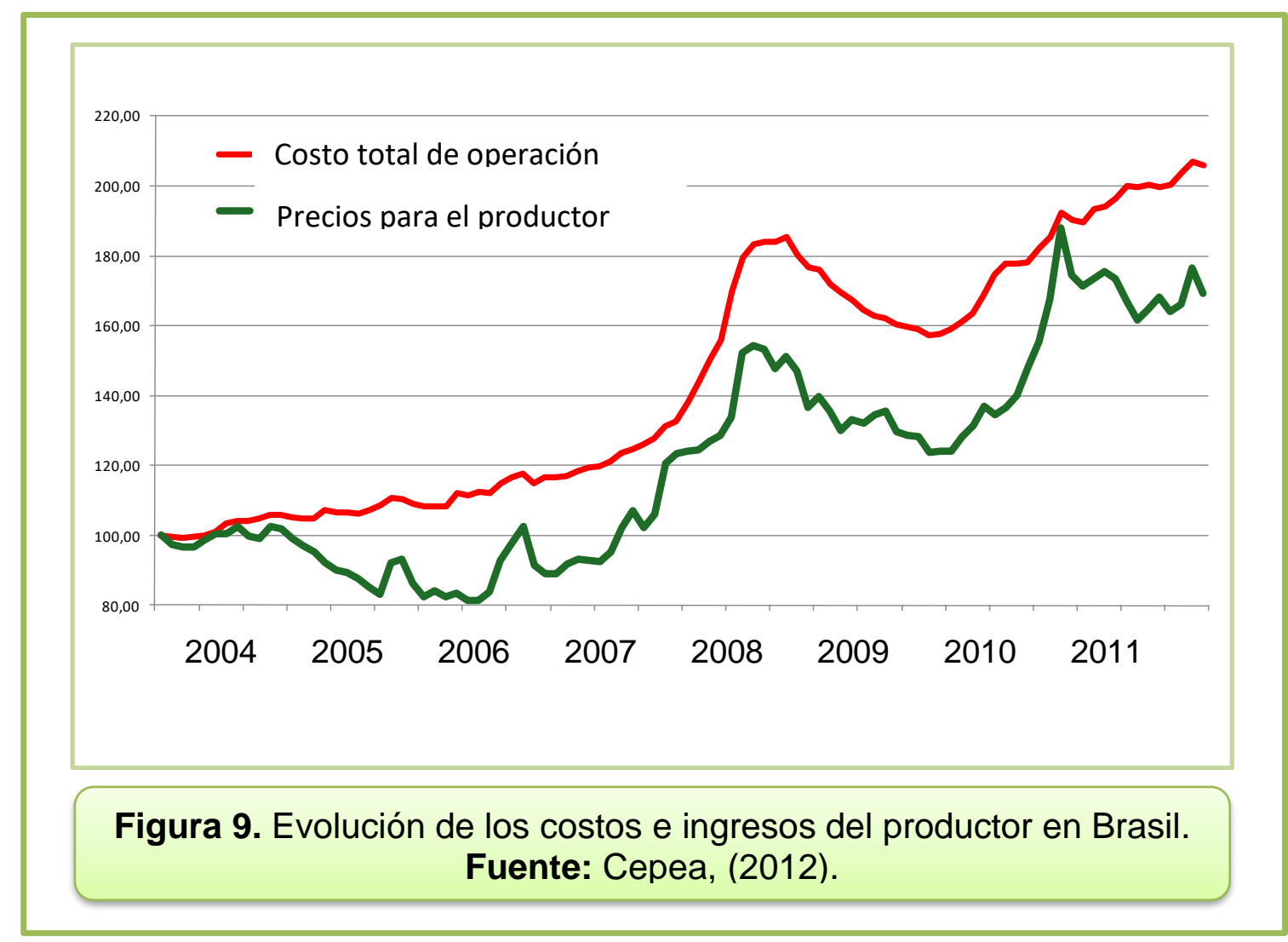

Comparando el costo de producción con el de otros países, la situación brasilera es mejor, donde se comparan 20 países, el nombre de los hatos es definido por la combinación del país y el número de animales vendidos por año, ejemplo, BR-140, hato de Brasil, que vende 140 bovinos por año, siendo Brasil y Argentina los de menor costo de producción de ganado de carne en el mundo, lo que muestra la competitividad del sector en América del Sur. En este aspecto hay mucho por mejorar en Brasil y América del sur, el indicador, ternero destetado / vaca, en Sur América, principalmente Brasil tiene un bajo índice, debido al poco uso de suplementación mineral y al alto costo de pastaje, observándose que los hatos de Argentina, Brasil, Colombia y Australia poseen los peores índices en procesos de producción, mercado externo e interno (Agribenchmark, 2011) (Tabla, 3).

\section{PERSPECTIVAS}

El futuro del mercado de las carnes y el agronegocio brasilero es promisorio, debido principalmente a la disponibilidad de tierra y agua en su territorio, también se suma a este hecho la estabilidad económica y política que favorece la inversión 
extranjera y nacional. Brasil tiene abundante disponibilidad de tierra cultivable (Figura 10), considerando la Unión Europea, Rusia y Congo, donde en las dos primeras naciones el clima impide la producción de granos en dos cosechas y la tercera tiene bajo volumen hídrico. En Brasil hay dos caminos a seguir, acelerar la inversión en capital humano, en nuevas tecnologías y principalmente en infraestructura (puertos, depósitos de almacenamiento y carreteras), además de trabajar en asuntos tributarios, que en muchos casos hace que los productos nacionales pierdan competitividad. Se observa perspectiva de crecimiento de la producción, consumo y excedentes en los próximos años para carnes y pescado (Tabla 4).

Tabla 3. Producción, exportación, importación, consumo interno y consumo per cápita de carne bovina en Brasil en los años de 1999-2011.

\begin{tabular}{|cccccc|}
\hline Año & $\begin{array}{c}\text { Producción } \\
\text { (Toneladas) }\end{array}$ & $\begin{array}{c}\text { Exportaciones } \\
\text { (Toneladas) }\end{array}$ & (Toneladas) & $\begin{array}{c}\text { Consumo } \\
\text { Interno } \\
\text { (Toneladas) }\end{array}$ & $\begin{array}{c}\text { Consumo } \\
\text { Percapita } \\
\text { (kg) }\end{array}$ \\
\hline 1999 & 6.270 .00 & 559.90 & 83.20 & 5.793 .30 & 34.33 \\
\hline 2000 & 6.650 .00 & 591.90 & 99.90 & 6.158 .00 & 35.95 \\
\hline 2001 & 6.900 .00 & 858.30 & 49.30 & 6.091 .00 & 35.04 \\
\hline 2002 & 7.300 .00 & 1.006 .00 & 100.70 & 6.394 .70 & 36.25 \\
2003 & 7.700 .00 & 1.300 .80 & 63.70 & 6.462 .90 & 36.11 \\
\hline 2004 & 8.350 .00 & 1.854 .40 & 53.30 & 6.548 .90 & 36.06 \\
\hline 2005 & 8.775 .86 & 2.197 .60 & 49.20 & 6.627 .46 & 35.98 \\
\hline 2006 & 9.052 .68 & 2.200 .00 & 28.50 & 6.881 .18 & 36.84 \\
\hline 2007 & 9.296 .73 & 2.350 .00 & 28.00 & 6.974 .73 & 36.84 \\
\hline 2008 & 9.011 .30 & 2.163 .31 & 25.80 & 6.873 .79 & 36.14 \\
\hline 2009 & 9.180 .00 & 1.924 .40 & 25.00 & 7.280 .60 & 37.77 \\
\hline 2010 & 9.445 .40 & 1.863 .14 & 27.50 & 7.609 .76 & 39.90 \\
\hline 2011 & 9.964 .90 & 1.913 .37 & 27.00 & 7.879 .32 & 14.42 \\
\hline
\end{tabular}

Fuente: (Agribenchmark, 2011).

La tasa de crecimiento en la producción de carne bovina del PIB fue establecida por el Banco central de Brasil, en los últimos años en 3,57\%, se nota que habrá excedentes de producción, en el caso que la misma permanezca en ese nivel y el consumo crezca correlacionado con el PIB estimado, lo que implicará una búsqueda de mercado para la venta de carne, o los precios nacionales sufrirán presión hacia la baja. 


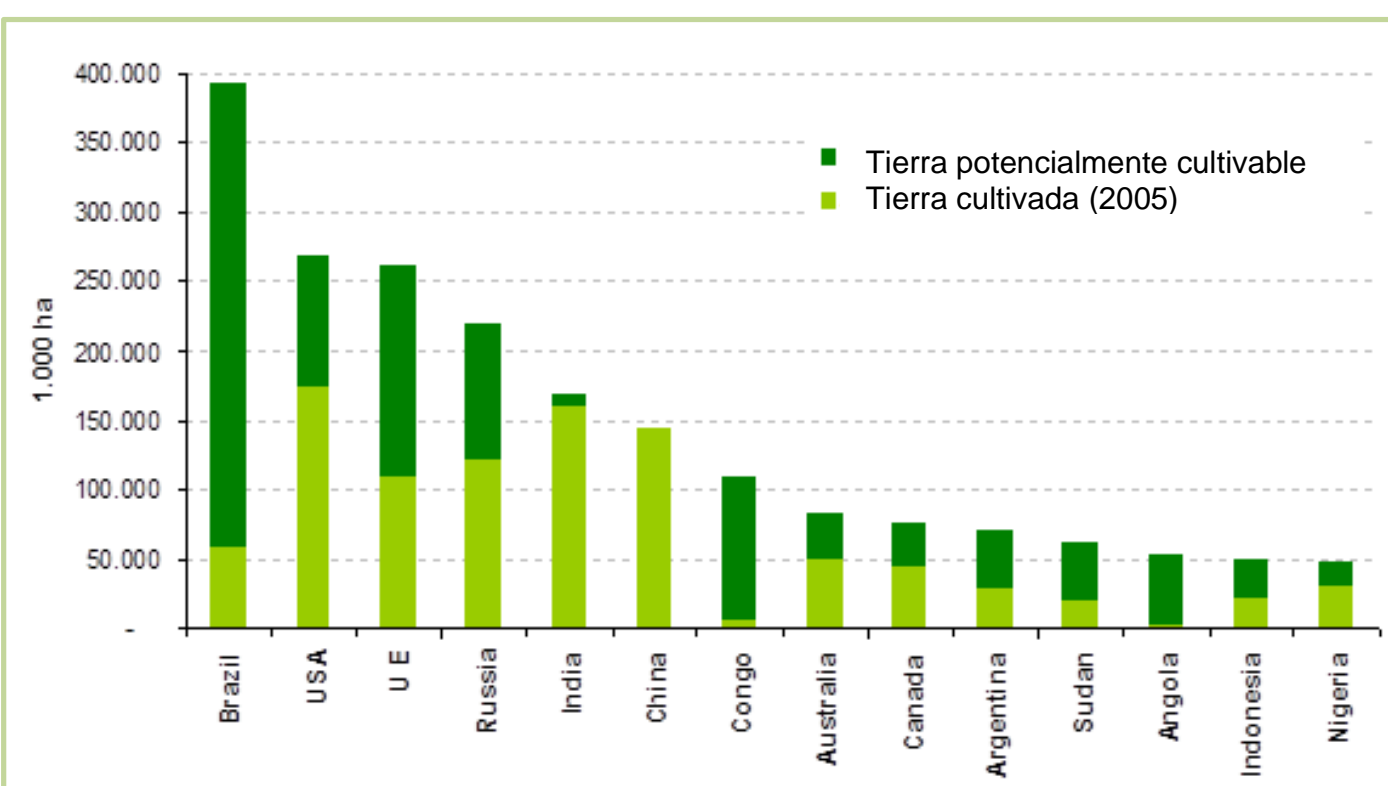

Figura 10. Disponibilidad de tierra cultivable y tierra en uso en algunos países.

Fuente: FAO, (2012).

Tabla 4. Estimativa de producción, consumo y excedentes de carne bovina para los años de 2012 a 2016

\begin{tabular}{ccccc}
\hline Años & $\begin{array}{c}\text { Tasa de } \\
\text { crecimiento } \\
\text { PIB \% }\end{array}$ & $\begin{array}{c}\text { Tasa de crecimiento de la Producción } \\
\text { Producción }\end{array}$ & $\begin{array}{c}\text { Consumo } \\
\text { (toneladas) }\end{array}$ & $\begin{array}{c}\text { Excedentes } \\
\text { (toneladas) }\end{array}$ \\
\hline 2012 & 3.22 & $10.436 .751,44$ & $8.245 .139,44$ & $2.191 .612,52$ \\
2013 & 4.30 & $10.868 .833,50$ & $8.489 .962,33$ & $2.378 .871,17$ \\
2014 & 4.00 & $11.318 .803,20$ & $8.733 .470,34$ & $2.585 .333,00$ \\
2015 & 4.00 & $11.787 .401,66$ & $8.983 .962,34$ & $2.803 .439,31$ \\
2016 & 4.00 & $12.275 .400,09$ & $9.241 .639,06$ & $3.033 .761,03$ \\
\hline
\end{tabular}

Fuente: Autores

\section{BIBLIOGRAFÍA}

1. AgriBenchmark. Beef Report 2011. Recuperado Acesso 20 abrril 2012. Disponible En: http://www.agribenchmark.org

2. Andrade D.R.; Yasui G.S. O manejo da reprodução natural e artificial e sua importância na produção de peixes no Brasil. Revista Brasileira de Reprodução Animal. 27 (2): 166-172, 2003.

3. Associação Brasileira das Indústrias Exportadoras de Carne. Estatísticas. Recuperado 19 abril 2012. Disponible En: http://www.abiec.com.br/ 
4. Barros R. P.; Mendonça R. A evolução do bem-estar, pobreza e desigualdade no Brasil ao longo das últimas três décadas: 1960/90. Pesquisa e Planejamento Econômico, Rio de Janeiro, 25 (1): 115-164, 1995.

5. Brasil Foods - BRFoods. Investidores. Recuperado 05 mayo 2012. Disponible En: http://www.brasilfoods.com/ri/

6. Carvalho T. B. de. Estudo da elasticidade-renda da demanda de carne bovina, suína e de frango no Brasil. Dissertação de Mestrado ESALQ/USP. 88 p. 2007.

7. Carvalho T. B. de. Elasticidade-renda da demanda de carne bovina, suína e de frango no Brasil. En: XLIX Congresso da Sociedade Brasileira de Economia e Sociologia Rural, 2011, Belo Horizonte. 2011.

8. Centro de Estudos Avançados em Economia Aplicada. Indicadores de preços. Recuperado 02 mayo 2012. Disponible En: http://www.cepea.esalq.usp.br/

9. Confederação da Agricultura e Pecuária do Brasil. Publicações: indicadores rurais. Recuperado 02 abril 2012. Disponible En: http://www.canaldoprodutor.com.br/instituto-cna

10. Food and Agriculture Organization of the United Nations - FAO. Estatísticas. Recuperado 19 abril 2012. Disponible En: http://www.fao.org/

11. Fundo Monetário Internacional - FMI. World Economic Outlook (WEO). $\begin{array}{lllll}\text { Recuperado } 26 & 2012 . & \text { abril }\end{array}$ http://www.imf.org/external/index.htm

12. Instituto Brasileiro de Geografia e Estatística. Pesquisa de orçamentos familiares 2008 - 2009. Recuperado 15 abril 2012. Disponible En: http://www.sidra.ibge.gov.br/bda/pesquisas/pof/

13. JBS - Grupo JBS Friboi. Relação com Investidores. Recuperado 05 mayo 2012. Disponible En: http://www.jbs.com.br/ri/

14. Jornal O Estado de São Paulo. Edição 23 abr. 2012.

15. GRUPO MARFRIG. Investidores. Recuperado 05 mayo 2012. Disponible En: http://ri.marfrig.com.br/port/governanca/composicao.asp

16. Martins E. Variações no consumo de alimentos no Brasil de 1974/75 a 1987/88. 1998. Dissertação Mestrado em Economia Aplicada - Escola Superior de Agricultura "Luiz de Queiroz", Universidade de São Paulo, Piracicaba, 117 p. 1998.

17. MINERVA. Relação com Investidores. Recuperado 05 mayo 2012. Disponible En: http://www.minerva.ind.br/ri/index.htm

18. Pinazza L. A., Araújo N. B. Agricultura na virada do século $X X$ : visão agribusiness. São Paulo: Globo S.A., 166 p. 1993. 